EPJ Web of Conferences 70, 00026 (2014)

DOI: $10.1051 /$ epjconf/ 20147000026

(C) Owned by the authors, published by EDP Sciences, 2014

\title{
AMS-02 on the International Space Station
}

\author{
Francesca R. Spada ${ }^{1, a}$ \\ ${ }^{1}$ Istituto Nazionale di Fisica Nucleare - P.le Aldo Moro 2 - 00185 Rome, Italy
}

\begin{abstract}
AMS-02 is a multipurpose magnetic spectrometer that will operate on the International Space Station for at least two decades. The detector is designed to measure with very high accuracy the composition of cosmic rays near Earth.

With a large acceptance, an intense magnetic field from a permanent magnet and a very efficient particle identification, AMS- 02 will provide the highest precision to date in cosmic rays measurements up to the $\mathrm{TeV}$ region, achieving a sensitivity to the existence of primordial antimatter in the cosmic rays of one part in a billion, and providing the most complete information on the indirect observation of dark matter.

During the first year in space, several billion events have been recorded. The flight operation, the detector performance, together with early results and perspective for physics measurements are reported.
\end{abstract}

\section{Introduction}

Cosmic rays were discovered by Victor Hess in 1912. After one century, though, many open questions remain, concerning the origin, composition, acceleration mechanisms, propagation and confinement in the Galaxy of the leptons, nuclei and photons reaching Earth from outer space.

Moreover, we have now learned that a deeper understanding of cosmic rays properties could help to unveil apparently unrelated puzzles, such as the nature of dark matter, the apparent absence of antimatter in the explored Universe, and the presence of exotic forms of matter.

The Alpha Magnetic Spectrometer (AMS-02) is a particle physics detector designed to measure charged cosmic ray spectra up to the $\mathrm{TeV}$ region, with a high-energy photon detection capability up to few hundred $\mathrm{GeV}$. The spectrometer is characterized by a large acceptance, long duration and state-of-the-art particle identification techniques.

The detector, quite large by space standards, and exploiting technologies developed for highenergy particle physics adapted to the harsh space environment, consists of a seven-layer Silicon Tracker inside a large permanent magnet plus two layers outside; a Transition Radiation Detector and a 3D-Electromagnetic Calorimeter that allow for electron, positron and photon identification; a Time of Flight scintillating system and a Ring Image Cherenkov detector that perform independent velocity measurements.

On May 19th, 2011 the Endeavour Shuttle handed AMS-02 to the robotic arm of the International Space Station for the installation, and the detector has since collected billions of cosmic ray events.

ae-mail: francesca.spada@cern.ch

This is an Open Access article distributed under the terms of the Creative Commons Attribution License 2.0, which permits unrestricted use, distribution, and reproduction in any medium, provided the original work is properly cited. 


\section{The physics of AMS-02}

AMS-02 is designed to address open questions shared by high-energy physics, astrophysics and cosmology, by performing a high-precision, large-statistics and long-duration study of cosmic nuclei, elementary charged particles and $\gamma$-rays, with unprecedent energy resolution up to the TeV region.

\subsection{The Standard Cosmological Model}

Precision measurements of cosmological quantities, such as the Cosmic Microwave Background temperature and polarization [1-3] and the Supernova luminosity-to-distance relationship, together with Large Scale Structure and structure formation studies [4, 5], rather recently allowed to build a solid and reliable Standard Cosmological Model.

According to the model, our universe is spatially flat, homogeneous and isotropic at large scales, and consists of ordinary matter and radiation only for a $4.5 \%$ : as shown in figure 1 , a considerable fraction of the total matter (23\%) is in the form of cold dark matter. The largest fraction (73\%) consists of dark energy, acting like a cosmological constant, whose origin is unknown [6].

In addition to this, independent measurements show that the antimatter content of the universe appears to be only $10^{-6}$ of the matter content [7].

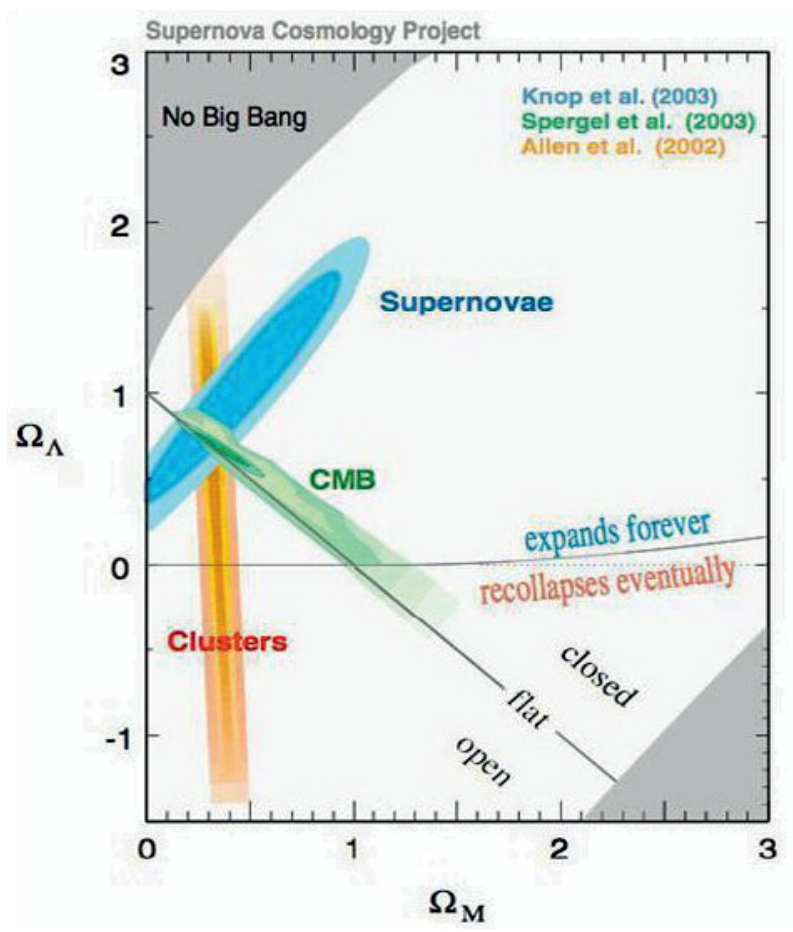

Figure 1. Independent measurements of several cosmological quantities all point in the direction of a universe composed mostly of dark matter and dark energy. 


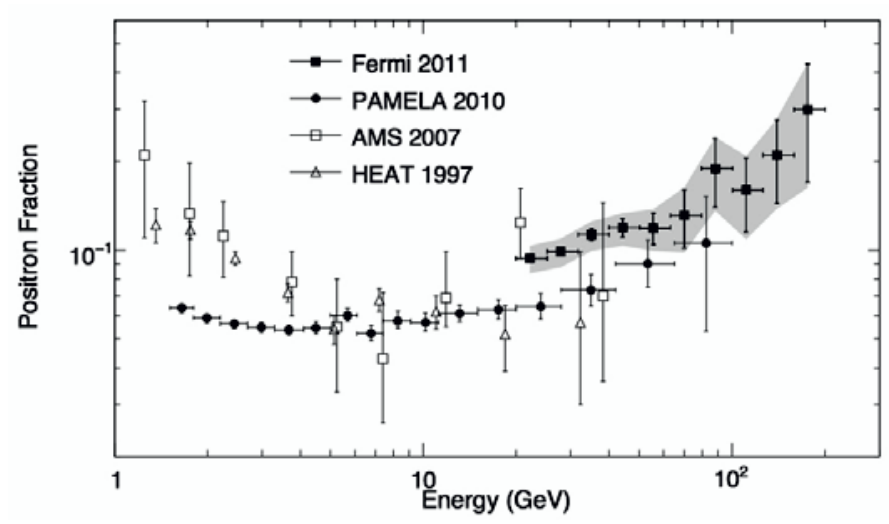

Figure 2. Fermi [10], PAMELA [11] and other recent measurements of the positron fraction. The AMS-01 [7, 12], Fermi and PAMELA results are from space-born experiments.

\subsection{Indirect search for dark matter}

AMS-02 can give a substantial contribution to the understanding of the nature of dark matter through indirect observation: the interactions of a dark matter candidate, such as a stable weakly interacting massive particle (WIMP), will appear as unexpected features in the energy spectra of particles such as electrons, positrons, protons, antiprotons and photons.

Existing measurements of the positron fraction (see figure 2) have in some cases shown deviations from standard model expectations which are consistent with supersymmetric dark matter, but controversial within the available statistics $[8,9]$.

Thanks to its excellent particle identification capabilities, AMS-02 is able to measure the positron spectrum in particular, but in general all the interesting fluxes, simultaneously and independently, thus enhancing the reliability of a potential discovery, with substantially improved precision for background and signal.

\subsection{Direct search for antimatter}

In the Big Bang theory, matter and antimatter are created with equal abundances, and the disappearance of antimatter requires barion number violation and another source of CP violation. Antiparticles are indeed produced in collisions between high energy particles, and are observed in the cosmic rays. However, an anti-Helium nucleus has a very small probability of being produced in collisions: $\phi(\overline{H e}) / \phi(H e) \sim 10^{-6}-10^{-8}$. The detection of a anti-Helium nucleus in cosmic rays would be a clear indication of the existence of an antimatter area somewhere in the universe.

AMS-02 will collect about $10^{9}$ nuclei with energies up to $2 \mathrm{TeV}$, with sensitivity up to anti-Iron, thus improving of a factor $10^{3}$ the limit put by the precursor flight AMS-01 [7].

\subsection{More AMS-02 physics goals}

The capability of AMS-02 to measure the energy- and time-dependent fluxes of cosmic nuclei up to Iron, to an unprecedented degree of precision, will help to better understand the propagation models, 


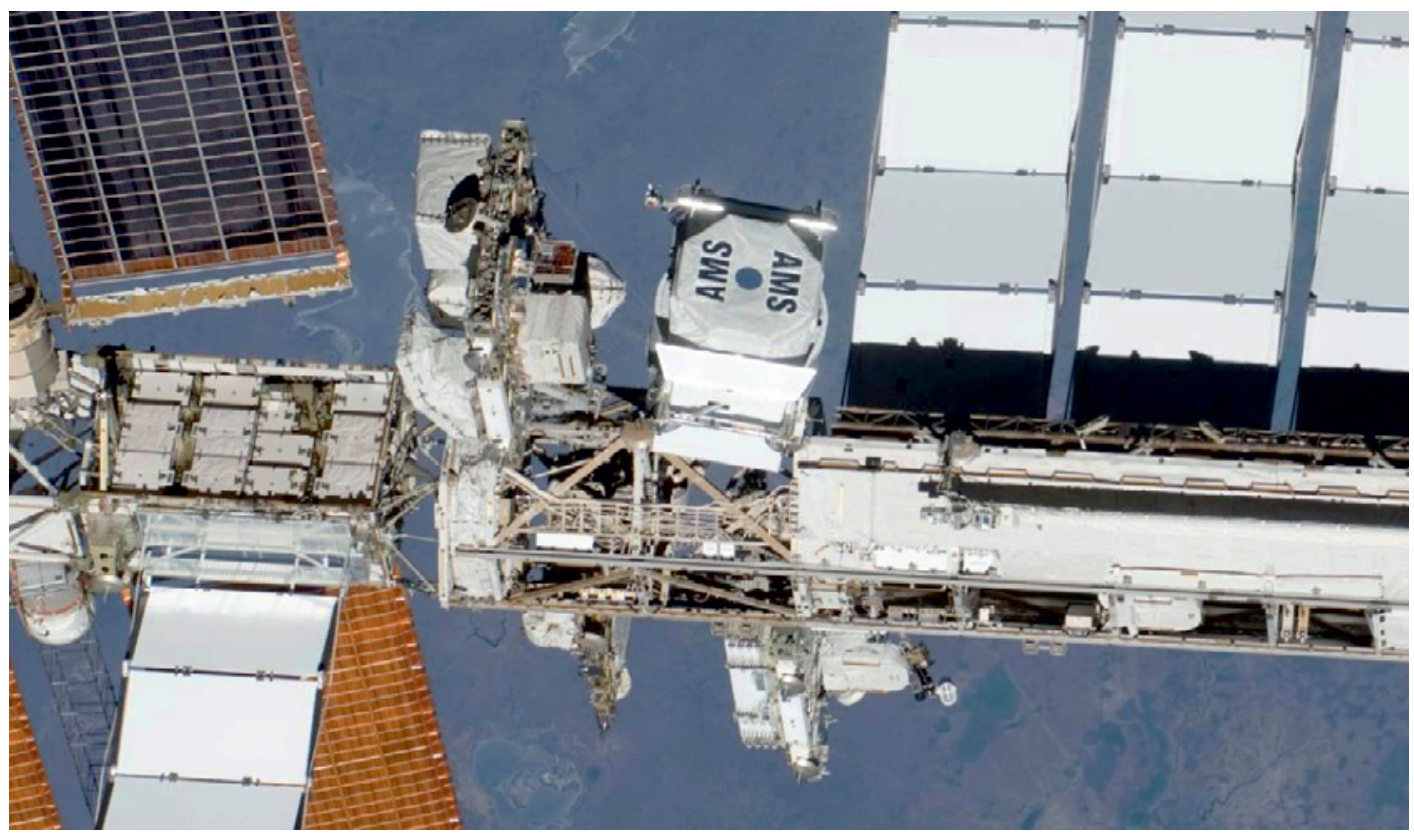

Figure 3. The AMS-02 experiment was installed in May 2011 on the main truss of the International Space Station.

the confinement mechanisms of cosmic rays in the Galaxy, and the intensity of the interactions with interstellar media.

Moreover, AMS-02 will explore the possible existence of new exotic forms of matter, such as strangelets, thanks to its excellent particle identification capability through precise mass and charge measurement.

The presence of a high-resolution electromagnetic calorimeter with very good tracking capabilities allows a precision measurements of the diffuse photon flux as well as the identification of local sources of high-energy $\gamma$-rays.

Finally, thanks to its sensitivity to low-energy (i.e. a few hundreds MeV) particles, AMS-02 can address several solar physics items and, in particular, measure the solar modulation of the cosmic rays spectrum over one entire solar cycle (about 11 years).

\section{The AMS-02 detector}

AMS-02 is a particle spectrometer with large acceptance, large momentum range and efficient particle identification, designed to provide high statistics spectra of charged particles with nuclei and isotope separation, as well as $\gamma$-ray measurements. Figure 3 shows the experiment installed in its location on the main truss of the International Space Station.

From the point of view of "mission success", the operation of a detector in the rough and unwelcoming outer space environment represents a challenge from the point of view of the design, of the selection of components and materials that ensure the reliability of the instruments, since no access is possible once the detector is installed. 


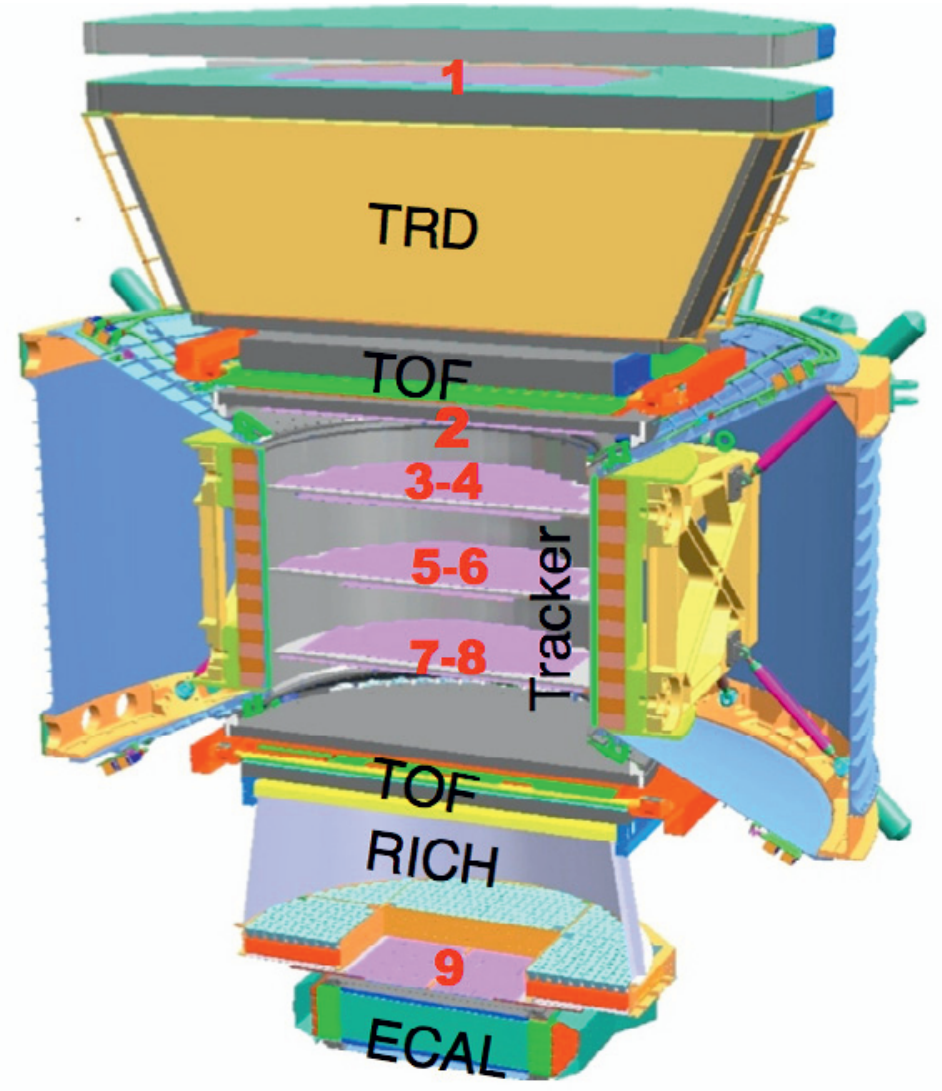

Figure 4. The AMS-02 experiment on the International Space Station. It consists of a silicon tracker installed inside a permanent magnet with two additional external planes; a 20 layer fibre fleece $\mathrm{Xe} / \mathrm{CO}_{2}$ proportional wire straw tube Transition Radiation Detector (TRD) on top; a four-layer Time of Flight (TOF); a Ring Imaging aerogel/NaF Cherenkov detector (RICH); a lead/scintillating fibre sandwich 3D sampling calorimeter (ECAL).

The system has to withstand the launch stress (vibration and depressurization), and has to operate stably in vacuum, at temperatures that oscillate between exremely low and very high values, in the presence of ionizing radiation and heavy ions, having at the same time strict constraints on the total weight, dimensions and power consumption allowed. The detector, shown in figure 4, measures 5 by 4 by 3 square meters, for a $7.5 \mathrm{t}$ weight.

AMS-02 is equipped with a large Nd-Fe-B permanent magnet providing a $1.4 \mathrm{kG}$ magnetic field, and five main subdetectors for the precise measurement of the particle mass, momentum, energy and charge. The redundancy of the measurement of velocity and charge guarantees an efficient particle identification and background rejection. AMS-02 is composed by:

a. the Time of Flight (TOF), consisting of four planes of plastic scintillators located above and below the permanent magnet. Each scintillator pad is read by two independent photomultipliers placed at its opposite ends. The TOF provides the fast trigger of the experiment and measures the particle velocity with a $\Delta \beta / \beta \sim 4 \%$ for protons, and the charge $\mathrm{Z}$ up to iron [13]; 
b. the Silicon Tracker [14], made of nine layers of silicon microstrip detectors mounted on five carbon fiber planes, for a total active area of about $6.4 \mathrm{~m}^{2}$. Simultaneous measurement of position and energy loss in silicon are performed along the charged particle trajectory in each layer, with a $10 \mu \mathrm{m}$ spatial resolution for singly charged particles, going down to $\sim 6 \mu \mathrm{m}$ for $\mathrm{Z}>1$. The low noise and wide dynamic range of the silicon readout electronics allow to exploit the energy loss measurements to determine the particle absolute charge for nuclei up to iron [15]. The rigidity and the charge sign are measured up to $2 \mathrm{TV}$, with a resolution $\sigma R / R \sim 2.5 \%$ up to $\mathrm{O}(100) \mathrm{GV}$;

c. the Transition Radiation Detector (TRD), consisting of 5248 straw tubes of $6 \mathrm{~mm}$ diameter packed in 328 modules and arranged in 20 layers, each interleaved with a $20 \mathrm{~mm}$ thick fleece radiator of $10 \mu \mathrm{m}$ polyetilene/polypropylene fibres with a density of $0.06 \mathrm{~g} / \mathrm{cm}^{3}$. The kapton straw tubes are filled with a $\mathrm{Xe} / \mathrm{CO}_{2}$ [90:10] gas mixture that efficiently captures the transition radiation generated in the radiator [16]. The $\mathrm{CO}_{2}$ tends to diffuse through the straw tube walls: with $5 \mathrm{~kg}$ of $\mathrm{CO}_{2}$ onboard, the measured leak rate of $5 \mu \mathrm{g} / \mathrm{s}$ corresponds to a lifetime of 30 years in space [17];

d. the Ring Imaging Cherenkov (RICH), a proximity focusing device with a dual solid radiator configuration on the top, an expansion height of $46.9 \mathrm{~cm}$ and, at the bottom, a matrix of 680 multipixelized photon readout cells. The central radiator is sodium fluoride with a refractive index of 1.33 , encircled by an aerogel radiator with a refractive index of 1.05 . The Cherenkov photons are detected by an array of 10880 photosensors. To reduce lateral losses, the expansion volume is surrounded by a high reflectivity mirror with the shape of a truncated cone. The RICH provides $\mathrm{Z}$ measurement up to iron and velocity with $\Delta \beta / \beta \sim 0.1 / Z \%$, allowing for isotope separation in the kinetic energy range from $0.5 \mathrm{GeV} / \mathrm{n}$ to $10 \mathrm{GeV} / \mathrm{n}$ for $\mathrm{A}=10$ [18];

e. the Electromagnetic Calorimeter (ECAL), consisting of lead and scintillating fibers with an active area of $648 \times 648 \mathrm{~mm}^{2}$ and a thickness of $166.5 \mathrm{~mm}$, corresponding to 17 radiation lengths, divided in 9 superlayers. The 3D imaging capability is obtained by stacking superlayers with fibers alternatively parallel to the $\mathrm{X}$ and $\mathrm{Y}$ axes. The ECAL gives a standalone trigger for photons and identifies electromagnetic showers, measuring the energy of electromagnetic particles with a resolution $\sigma_{E} \sim 3 \%$ above $10 \mathrm{GeV}$ [19].

AMS-02 is also equipped with 16 plastic scintillator paddles of Anti Coincidence Counters (ACC), that ensure that only particles passing the magnet aperture are accepted. The paddles form a cylinder of $109 \mathrm{~cm}$ diameter and are located along the inner surface of the magnet.

\section{Detector operation in space}

On May 19, 2011 the AMS-02 installation was completed at 5:15 AM. The data taking started at 9:35 AM. Since then, the detector, with its 300,000 electronics channels, 650 computers, 1100 thermal sensors and 400 thermostats, has worked continuously.

The Payload Operation Control Centre (POCC) at CERN was operative few months after the AMS-02 installation on the ISS, after a brief phase in which the experiment was controlled from NASA Johnson Space Center (JSC) in Houston, TX. The POCC is in permanent connection with the ISS, the JSC and the NASA Marshall Space Flight Center in Huntsville, AL where both commands sent to AMS-02, and data coming back are handled.

The POCC is operational $24 \mathrm{~h} /$ day, and supervises the payload operation and monitors continuously the detector performance. 


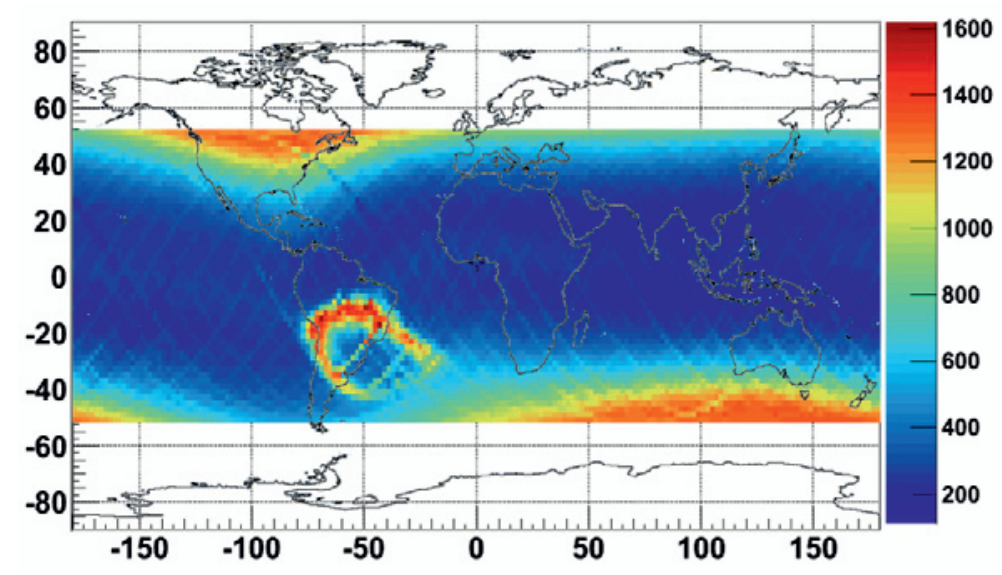

Figure 5. Data acquisition rate of AMS-02 varying from 200 to about $2000 \mathrm{~Hz}$ over one orbit. The rate is higher towards the poles and in correspondence of the South Atlantic Anomaly, and decreases along the Equator.

\subsection{On-orbit thermal control}

The control of the payload temperature conditions is a crucial task since the thermal environment on ISS is constantly changing due to the solar $\beta$ angle (defined as the angle between the ISS orbital plane and the solar vector), the ISS attitude, the position of the ISS radiators and solar arrays, and the Earth albedo.

The $\beta$ angle changes constantly (about $4.5^{\circ} \mathrm{C}$ /day) from $-75^{\circ} \mathrm{C}$ to $+75^{\circ} \mathrm{C}$ due to the precession of the ISS orbit and the rotation of the Earth around the Sun. At extreme $\beta$ values, AMS-02 is partly illuminated and partly in shade, leading to large temperature gradients.

The changing orientation (or attitude) of the ISS affects the direction of the sunlight hitting the payload, again causing temperature variations.

A sophisticated thermal system, including a large two-phase $\mathrm{CO}_{2}$ cooling system for the silicon tracker, protects the experiment from the sharp temperature changes. Heaters are placed in key locations such as the TRD gas circuit, and can be activated if the local temperature is getting too close to the gas freezing points. However, temperature conditions out of the safe range can easily damage or destroy the detector, thus all functional parameters are constantly monitored. In case of need, actions are taken in the POCC to restore safe conditions.

\subsection{Data acquisition and detector performance}

The Science Operation Center (SOC) at CERN provides fast processing of data upon reception. The acquisition rate varies from 200 to $2000 \mathrm{~Hz}$ over the orbit (see figure 5), with an average rate of $700 \mathrm{~Hz}$ and $85 \%$ DAQ efficiency.

AMS-02 collected over 16 billion events over the first 12 months, corresponding to 35 TB raw data. The particle collected so far have been used to carefully calibrate the subdetectors.

An example of the excellent results achieved is the effect of alignment and calibration on the TRD performance. Due to temperature variations, the TRD is moving on top of the inner tracker up to $1 \mathrm{~mm}$ (figure 6, top-left). Cosmic protons were used to align the straw modules to an accuracy of $0.04 \mathrm{~mm}$ (figure 6, bottom-left). In addition to this, temperature, pressure, gas composition and HV 

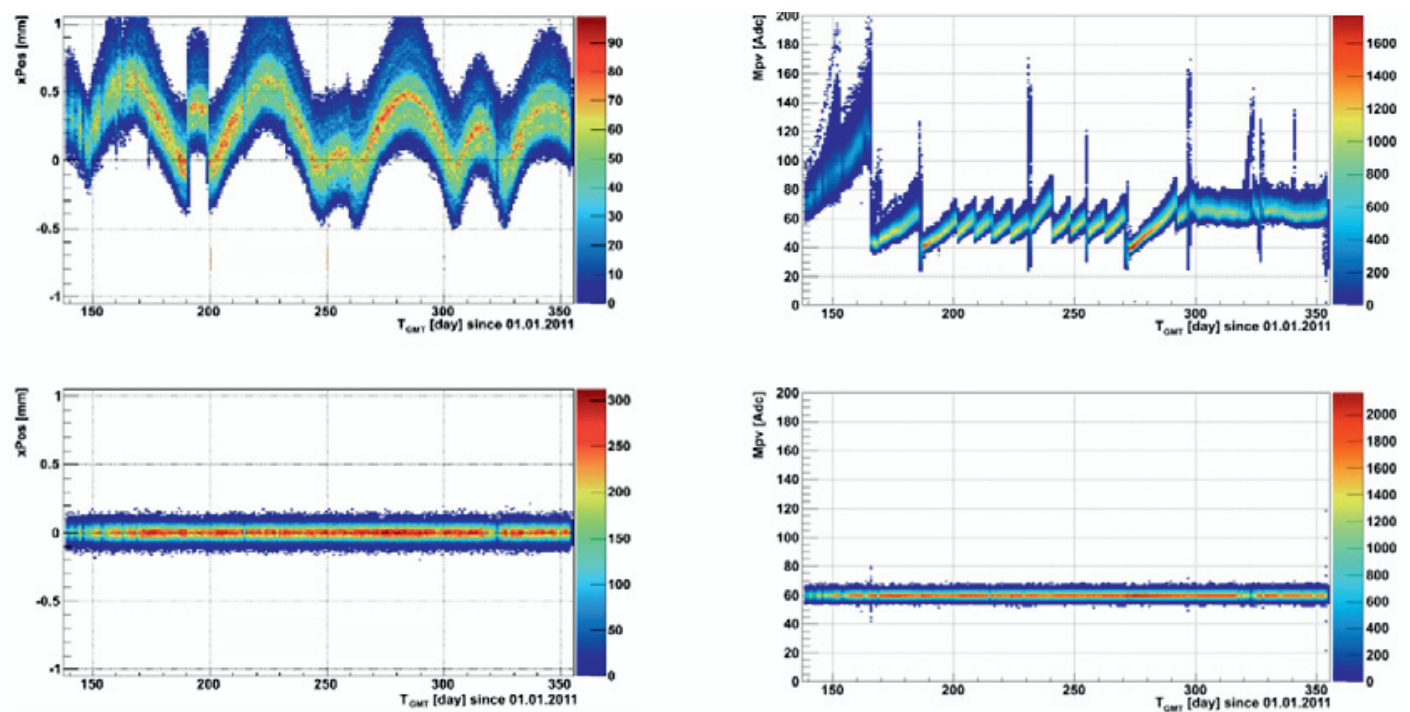

Figure 6. Top-left: TRD position shift over time before alignment; bottom-left: after alignment. Top-right: most probable value of the Landau peak of the TRD response over time before calibration; bottom-right: after calibration.

changes affect the gas gain and thus the TRD response over time (figure 6 top-right). Again protons were used to calibrate the detector response to a $3 \%$ accuracy (figure 6 bottom-right).

One of the key features of AMS-02 is the redundancy of the velocity and charge measurement, crucial for unambiguous particle identification and background rejection. Figure 7 shows the potentialities of the combination of charge measurement from TOF, RICH and Tracker. The relative abundances of nuclei and isotopes in cosmic rays are essential for the understanding of their origin and propagation mechanisms.

\section{First results}

AMS-02 has already collected several nuclei with rigidities in the TeV range. In particular, the Helium abundance in cosmic rays already allowed the observation of the rate variation with the angle $\Theta_{M}$ between the Equator and the ISS position vector, as shown in figure 8.

On January 24, 2012 a solar flare occurred. AMS-02 was able to observe its effect, as shown in figure 9, that consisted, as expected, in an enhancement of the low-energy event rate, more evident in the equatorial region.

\section{Conclusions}

The Cosmos is the ultimate laboratory, since cosmic rays are observed with energies remarkably higher than those that can be reached at any accelerator.

AMS-02 is in space since May 2011 and all detectors are functioning as expected, and the DAQ is running in nominal conditions since the very early phases. The ground operations are running smoothly and the detector performances are being optimised. 

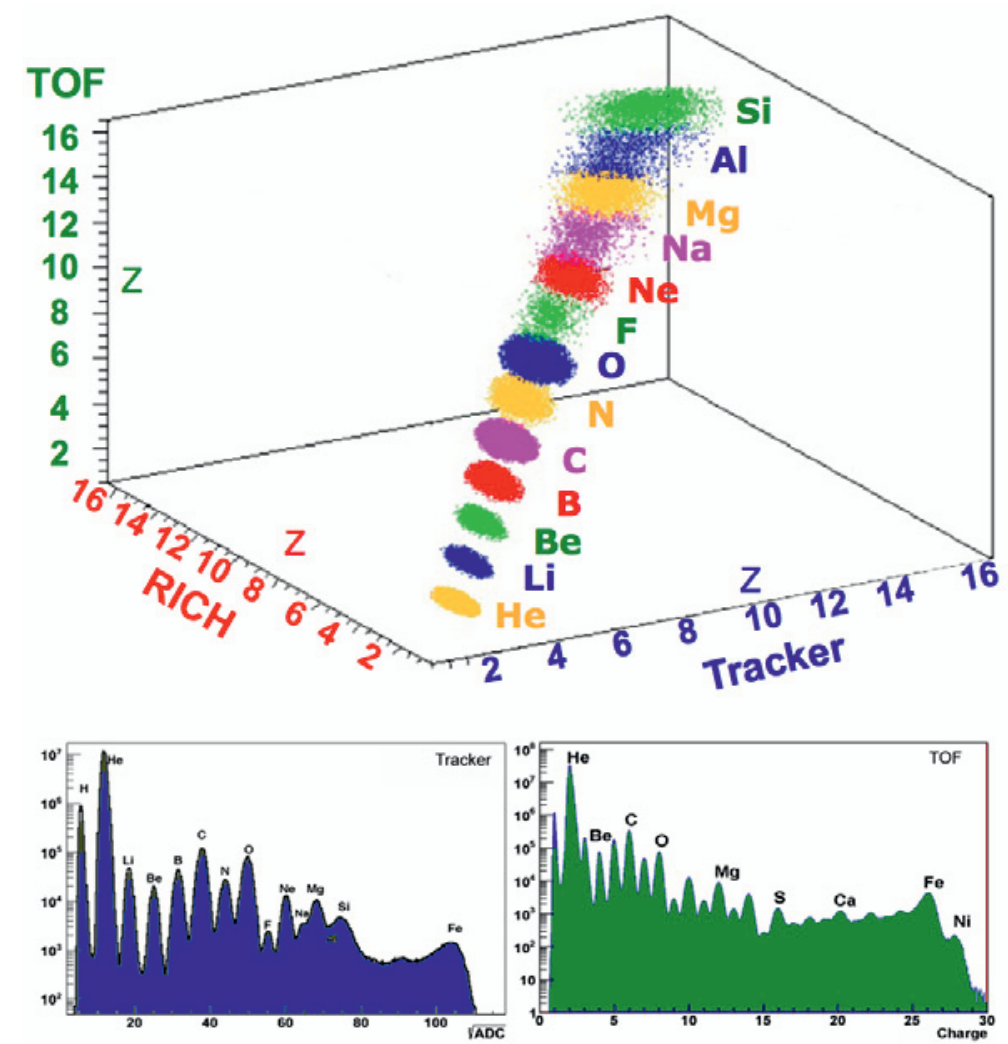

Figure 7. Top: combination of charge measurements from TOF, Tracker and RICH. Standalone tracker (bottom left) and TOF (bottom right) are sensitive to nuclei charges up to iron.

Already at this very early stage of operation the experiment is demonstrating its excellent capabilities. In two decades on board of the ISS, AMS-02 is going to collect about $1.5 \cdot 10^{10}$ events per year: this will provide unprecedent statistics that, together with the high sensitivity, guarantees a great discovery potential. 
EPJ Web of Conferences

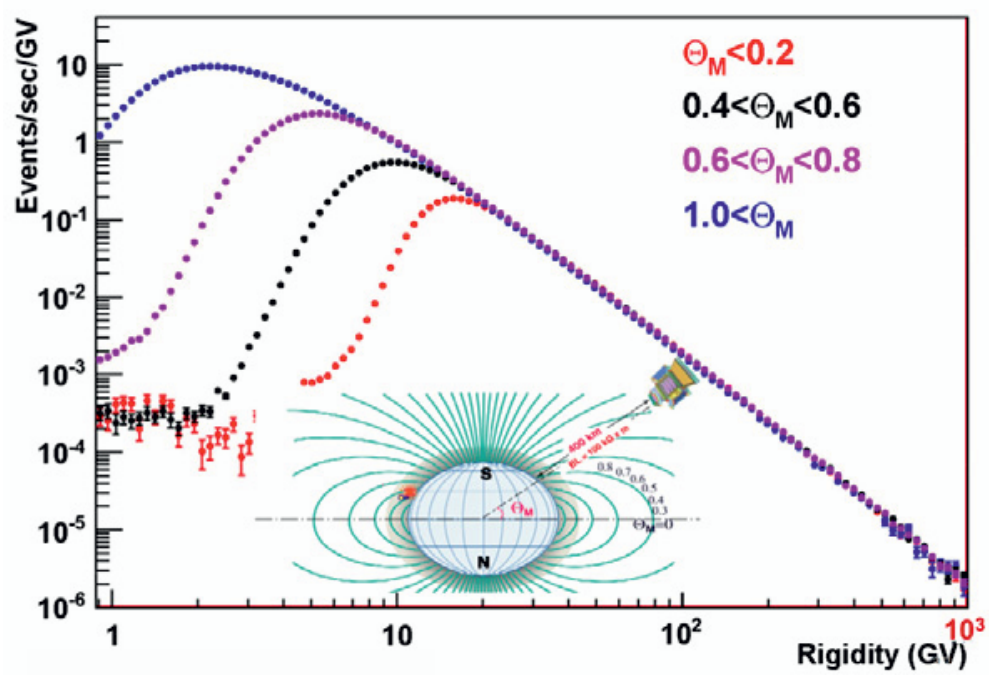

Figure 8. Helium rate at different values of $\Theta_{M}$. As shown in the inset, this is the angle between the Equator and the ISS position vector.

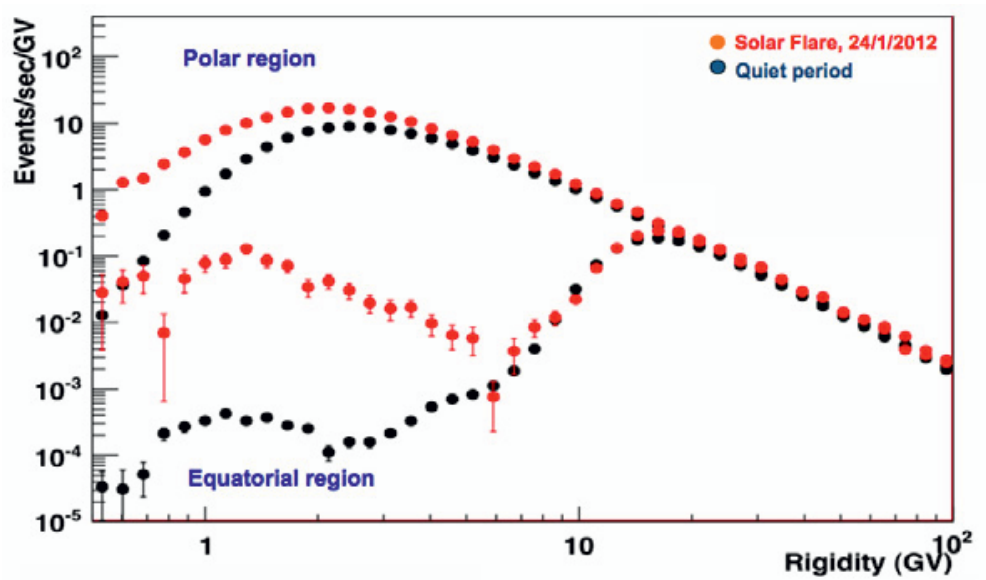

Figure 9. The effect of a solar flare on the Helium rate was an increased low-energy event rate. The effect is more evident in the equatorial region. 


\section{References}

[1] D. Spergel et al. (WMAP Collaboration), Astrophys.J.Suppl. 170, 377 (2007), astro-ph/0603449

[2] L. Page et al. (WMAP Collaboration), Astrophys.J.Suppl. 170, 335 (2007), astro-ph/0603450

[3] C. Netterfield et al. (Boomerang Collaboration), Astrophys.J. 571, 604 (2002), astro-ph/0104460

[4] A.G. Riess et al. (Supernova Search Team), Astrophys.J. 607, 665 (2004), astro-ph/0402512

[5] S. Perlmutter, M.S. Turner, M.J. White, Phys.Rev.Lett. 83, 670 (1999), astro-ph/9901052

[6] R.A. Knop et al. (Supernova Cosmology Project), Astrophys.J. 598, 102 (2003), astro-ph/0309368

[7] J. Alcaraz et al. (AMS Collaboration), Phys.Lett. B461, 387 (1999), hep-ex/0002048

[8] A.W. Strong, I.V. Moskalenko, O. Reimer, Astrophys.J. 613, 962 (2004), astro-ph/0406254

[9] W. de Boer, C. Sander, V. Zhukov, A. Gladyshev, D. Kazakov, Phys.Rev.Lett. 95, 209001 (2005), astro-ph/0602325

[10] M. Ackermann et al. (Fermi LAT Collaboration), Phys.Rev.Lett. 108, 011103 (2012), 1109.0521

[11] O. Adriani et al. (PAMELA Collaboration), Nature 458, 607 (2009), 0810 . 4995

[12] M. Aguilar et al. (AMS-01 Collaboration), Phys.Lett. B646, 145 (2007), astro-ph/0703154

[13] V. Bindi, D. Casadei, G. Castellini, F. Cindolo, A. Contin et al., Nucl.Instrum.Meth. A623, 968 (2010)

[14] G. Ambrosi, W. Burger, A. Oliva et al. (AMS-02 Collaboration), Nucl.Instrum.Meth. A617, 471 (2010)

[15] J. Alcaraz, B. Alpat, G. Ambrosi, P. Azzarello, R. Battiston et al., Nucl.Instrum.Meth. A593, $376(2008)$

[16] F. Hauler, W. de Boer, L. Jungermann, M. Schmanau, A. Bartoloni et al., IEEE Trans.Nucl.Sci. 51, 1365 (2004)

[17] F.R. Spada, Nucl.Instrum.Meth. A718, 440 (2013)

[18] M. Aguilar-Benitez, L. Arruda, F. Barao, G. Barreira, A. Barrau et al., Nucl.Instrum.Meth. A614, 237 (2010)

[19] L. Girard, C. Adloff, G. Coignet, C. Goy, R. Kossakowski et al., pp. 55-59 (2003) 